\title{
Electric dipole moment of light nuclei in chiral effective field theory
}

\author{
Alex Gnech ${ }^{1, *}$, Jordy de Vries ${ }^{2,3}$, Sachin Shain ${ }^{3}$, and Michele Viviani ${ }^{4}$ \\ ${ }^{1}$ Theory Center, Jefferson Lab, Newport News, Virginia 23606, USA \\ ${ }^{2}$ Amherst Center for Fundamental Interactions, Department of Physics, University of Massachusetts \\ Amherst, Amherst, MA 01003, USA \\ ${ }^{3}$ RIKEN BNL Research Center, Brookhaven National Laboratory, Upton, New York 11973-5000, USA \\ ${ }^{4}$ INFN-Pisa, 56127 Pisa, Italy
}

\begin{abstract}
.
$\mathrm{CP}$-violating interactions at quark level generate $\mathrm{CP}$-violating nuclear interactions and currents, which could be revealed by looking at the presence of a permanent nuclear electric dipole moment. Within the framework of chiral effective field theory, we discuss the derivation of the CP-violating nuclear potential up to next-to-next-to leading order $\left(\mathrm{N}^{2} \mathrm{LO}\right)$ and the preliminary results for the charge operator up to next-to leading order (NLO). Moreover, we introduce some renormalization argument which indicates that we need to promote the short-distance operator to the leading order (LO) in order to reabsorb the divergences generated by the one pion exchange. Finally, we present some selected numerical results for the electric dipole moments of ${ }^{2} \mathrm{H},{ }^{3} \mathrm{He}$ and ${ }^{3} \mathrm{H}$ discussing the systematic errors introduced by the truncation of the chiral expansion.
\end{abstract}

\section{Introduction}

The measurement of electric dipole moments (EDMs) of particles is the most promising observable for studying new sources of charge $(\mathrm{C})$ and parity $(\mathrm{P})$ violation $[1,2]$. The standard model (SM) has a natural source of CP violation in the Cabibbo-Kobayashi-Maskawa quark mixing matrix; however, it only induces EDMs through multiple electroweak loops and lead to immeasurably small values for EDMs [3,4]. Therefore, any non-vanishing EDM of a nuclear or an atomic system would highlight $\mathrm{CP}$ violating effects due to the so-far undiscovered quantum chromo dynamic (QCD) $\theta$-term or from beyond-the-SM (BSM) sources of CP violation. Current experimental EDM limits [5-7] constrain large set of CP-odd dimensionsix operators at the multi- TeV scale at the level of SM effective field theory (SMEFT), that translate in limits on BSM models.

The connection between the SMEFT and the EDMs of complex objects such as nuclei is not trivial. However, the combination of lattice QCD (LQCD) [8-10], chiral effective field theory $(\chi$ EFT) [11-13] and ab-initio nuclear calculation [14-16] permits to exploit this connection, improving significantly model-independent first principle calculations. The starting point is the SMEFT which permits to derive CP-violating operator that involves only light

\footnotetext{
*e-mail: agnech@jlab.org
} 
quarks, gluons and photons. Using then $\chi$ EFT it is possible to construct CP-violating Lagrangians where the relevant degrees of freedom are pions, nucleons and photons. Associated to each Lagrangian there is a low-energy constant (LEC) that take care of all the higher energy degrees of freedom. The LECs can be ideally calculated by LQCD. From the $\chi$ EFT Lagrangian is then possible to derive the CP-violating nuclear interactions and currents, which, in combination with modern few-body ab-initio techniques, can be used to compute the EDM of light nuclei. It is important to note that also the LECs have different chiral order based on the source of CP-violation [11, 12]. In this sense only several independent measurements of different nuclear EDMs can permit to fix all the LECs and then to distinguish between the various possible sources of $\mathrm{CP}$-violation.

The $\chi$ EFT approach is based on the observation that the chiral symmetry exhibited by QCD has a noticeable impact in the low-energy regime. Therefore, the CP-violating interactions among pions, nucleons and photons are constrained in order to reproduce the same behavior under chiral transformation that is given at the SMEFT level. Moreover, the $\chi$ EFT framework provides an expansion of nuclear amplitudes in terms of $p / \Lambda_{\chi}$ where $p \sim m_{\pi} \sim O(100 \mathrm{MeV})$ and $\Lambda_{\chi} \sim 4 \pi F_{\pi} \sim O(1 \mathrm{GeV})$ [17-19], where $F_{\pi} \simeq 92.4 \mathrm{MeV}$ is the pion decay constant.

EDMs of light nuclei were computed using CP-violating interactions that were derived up to $\mathrm{N}^{2} \mathrm{LO}[16,20]$. However, a full consistent calculation of the EDMs would require the development of the corresponding electromagnetic current (in term of the charge operator) up to the same order. As the best of our knowledge, within $\chi \mathrm{EFT}$, this work has been only partially carried out in Ref. [21], and only for the deuteron. Moreover, some recent results [22] showed that the Weinberg's power counting scheme, based on naive dimensional analysis (NDA) of the LECs, is not reliable in the nuclear sector for CP-violating interactions. Therefore, we need to reconsider the chiral order at which some of the interactions appear.

In this proceeding, after discussing some basic definitions for the computation of the EDM of nuclei in Section 2, we will briefly discuss the derivation of the CP-violating nuclear interactions and currents in Section 3. In this section we will also present the arguments contained in Ref. [22] in order to reconsider the NDA power counting scheme. Finally selected numerical results are provided in Section 4.

\section{Basics on nuclear EDMs}

The $\mathrm{EDM} d_{A}$ of a nucleus is given by the matrix element

$$
d_{A}=\lim _{q^{2} \rightarrow 0} \frac{1}{i q}\left\langle\psi^{A, J, J}|\rho(q)| \psi^{A, J, J}\right\rangle,
$$

where $q$ is the momenta carried by the exchanged photon, $\psi^{A, J, J}$ the wave function of the nucleus of mass $A$ with total angular momentum $J_{z}=J$ and $\rho(q)$ is the electromagnetic charge operator. The limit written in this way indicates that only the first multipole of the charge operator $(\mathrm{C} 1)$ is considered. In absence of the $\mathrm{CP}$-violating term this matrix element is zero due to the different parity generated by the $\mathrm{C} 1$ multipole.

In this matrix element the CP-violation can be introduced in two different ways. The first is through the wave function. The CP-violating interactions between pions and nucleons at the Hamiltonian level is expressed as a CP-violating term in the nuclear potential. Therefore, the solution of the Hamiltonian contains a CP-violating part, and the final wave function can be then written as

$$
\psi^{A, J, J}=\psi_{P C}^{A, J, J}+\psi_{\varnothing P}^{A, J, J},
$$


where with $P C$ we indicate the parity conserving and time-reversal conserving quantities and with $C P$ the $\mathrm{CP}$-violating ones. In this case the charge operator that will appear in the matrix element will be the standard PC charge operator. At leading order (LO) the only relevant operator correspond to the electric dipole moment operator it-self, namely

$$
\rho_{P C}^{\mathrm{LO}}(q)=i e \sum_{i=1}^{A} \frac{\left(1+\tau_{z}(i)\right)}{2} \boldsymbol{r}_{i} \cdot \boldsymbol{q}
$$

where $e$ is the electric charge, $\boldsymbol{r}_{i}$ the position of the nucleon $i$ and $\tau_{z}(i)$ the isospin projection along the $z$-axis of the nucleon $i$. The complete expression for the charge operator up to $\mathrm{N}^{3} \mathrm{LO}$ can be found in Ref. [23]. We will indicate this contribution to the nuclear EDM as $d_{A}^{V}$ and it is explicitly given by

$$
d_{A}^{V}=2 \times \lim _{q^{2} \rightarrow 0} \frac{1}{i q}\left\langle\psi_{P C}^{A, J, J}\left|\rho_{P C}(q)\right| \psi_{\text {CP }}^{A, J, J}\right\rangle .
$$

The second way, it is to introduce the CP-violation through the charge operator it-self. In this case only the $P C$ term of the wave function is needed since the CP-violating wave function would generate higher order contributions in the CP-violating LECs that can be discarded. The LO contribution of $\rho_{C \text { p }}(q)$ is given by

$$
\rho_{\text {CP }}^{\mathrm{LO}}(q)=i \sum_{i=1}^{A}\left(d_{n}\left(\frac{1-\tau_{z}(i)}{2}\right)+d_{p}\left(\frac{1+\tau_{z}(i)}{2}\right)\right) \boldsymbol{\sigma}_{i} \cdot \boldsymbol{q},
$$

where $\sigma$ indicates the Pauli matrices and $d_{n}$ and $d_{p}$ are the intrinsic EDM of the neutron and the proton respectively. The higher order terms of the charge operator up to NLO are discussed in Section 3. We can write this contribution to the EDM as

$$
d_{A}^{\rho}=\lim _{q^{2} \rightarrow 0} \frac{1}{i q}\left\langle\psi_{P C}^{A, J, J}\left|\rho_{C P}(q)\right| \psi_{P C}^{A, J, J}\right\rangle .
$$

The final nuclear EDM is then given by the sum of Eqs. (4) and (6), namely

$$
d_{A}=d_{A}^{V}+d_{A}^{\rho} .
$$

\section{CP-violating interaction and charge operator}

Starting from the chiral Lagrangians derived in Refs. [11, 12] it is possible to obtain the nuclear interaction using the time ordered perturbation theory (TOPT) [16] or the heavybarion (HB) expansion [20]. The TOPT diagrams which contributes to the nuclear potential order by order up to $\mathrm{N}^{2} \mathrm{LO}$ are given in Figure 1. The results are identical to the ones obtained by using the HB expansion except that the relativistic corrections are typically counted one order lower. Note that we do not considered at this level the different power counting that comes from the LECs them-selves that depends explicitly on the source of CP-violation at the fundamental level.

The explicit formula for the nuclear potential order by order can be found in Refs. [16, 20]. Here we briefly comment some details. In Figure 1 we separate the various diagrams on the base of the LEC that is attached to them. The LECs $g_{0}, g_{1}$ and $g_{2}$ present the typical topology of the one-pion exchange (OPE) at LO and two-pions exchange at $\mathrm{N}^{2} \mathrm{LO}$. A characteristic of the CP-violating interaction is the presence of a three-pions vertex that generate at NLO a new topology. At the same order also three-body forces arises due to the same vertex. 
By considering NDA the contact interactions appear at $\mathrm{N}^{2} \mathrm{LO}$. However, following the analysis of Ref. [22], the iteration of the OPE CP-violating term proportional to $g_{0}$ in the Lippmann-Schwinger equation (that is the analogous of solving the Hamiltonian) generate divergences that cannot be cured at LO, if not by promoting a counter-term (i.e. the contact interaction) at LO as well. In Figure 1 this is indicated by the red arrow. The same promotion is not necessary for the OPE proportional to $g_{1}$ [22]. There are no analysis about the OPE diagrams proportional to $g_{2}$ so far, even if the similar operator structure to the $g_{0}$ term suggest that the promotion of the relative counter-term at LO could be necessary. While for the numerical computation of the contribution of the single diagrams the change of the power counting has no impact, it has an enormous impact when the LECs are fitted to reproduce the experimental data.

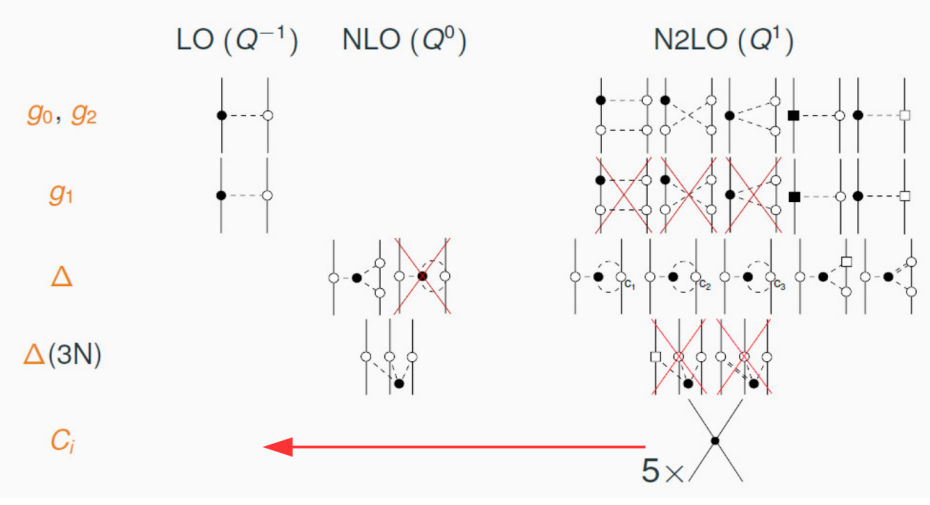

Figure 1. Diagrams contributing to the CP-violating nuclear potential for each different LEC following the NDA. The full (empty) circles indicates CP-violating (conserving) vertices at LO. The full (empty) squares indicates CP-violating (conserving) vertices at NLO. The double dashed lines indicate the second order in the pion propagator. Note that the red crossed diagrams cancel out when all the time ordered diagrams are summed. The red arrow indicate the necessity of promoting some of the contact interactions as counter-term at LO. See the text for more details.

In Figure 2 we present the preliminary results for the time ordered diagrams of the CPviolating charge operator up to NLO. As can be seen by inspecting the figure at LO we have the contribution of the single nucleon EDM (see Eq. (5)) and also of the OPE exchange with the photon attached to the pion on flight. However the time-order diagrams of this topology cancel out. Therefore, the first contributions beyond the single nucleon EDM come at NLO where we have again OPE with the photon in the nucleon-pion vertices or with the nucleon only. Note that there is a new LEC related to a photon-pion-nucleon CP-violating vertex. At the same order also different three-pion diagrams with a photon appear. The TPE and the contact diagrams that contribute to the charge current are instead at $\mathrm{N}^{2} \mathrm{LO}$. The explicit formula of the CP-violating charge operator will be object of a future publication [24]. 


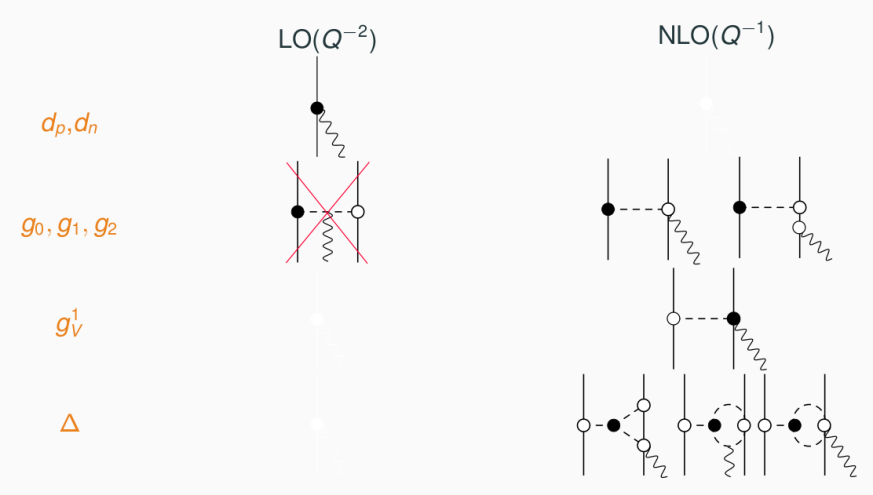

Figure 2. Diagrams contributing to the $\mathrm{CP}$-violating charge current for each different $\mathrm{CP}$-violating LEC. The full (empty) circles indicates CP-violating (conserving) vertices at LO. Note that the red crossed diagrams cancel out when all the time ordered diagrams are summed.

\section{Selected numerical results}

The EDM of nuclei can be then expressed as a product of the LECs with some numerical coefficients that contain the dynamic of the potential and the charge operator, namely

$$
\begin{aligned}
d_{A}= & g_{0}\left(a_{0}^{V}+a_{0}^{\rho}\right)+g_{1}\left(a_{1}^{V}+a_{1}^{\rho}\right)+g_{2}\left(a_{2}^{V}+a_{2}^{\rho}\right)+\Delta\left(a_{\Delta}^{V}+a_{\Delta}^{\rho}\right) \\
& +\sum_{i=1}^{5} C_{i}\left(A_{i}^{V}+A_{i}^{\rho}\right)+d_{p} a_{p}+d_{n} a_{n}+g_{V}^{1} a_{1 V}^{\rho},
\end{aligned}
$$

where the numerical coefficients $a_{i}^{V}$ are the contributions that come from Eq. (4) while the coefficients $a_{i}^{\rho}$ are the ones coming from Eq. (6). For now we only have results for the first ones, computed with only the LO charge operator (see Eq. (3)). While some tests showed that next orders corrections coming form the chiral expansion of the $P C$ charge operator are numerically very small and almost negligible, this is not true for the CP-violating charge operator. Therefore, a complete calculation where also the $a_{i}^{\rho}$ coefficients are computed is necessary to have a fully consistent description of nuclear EDMs.

In order to perform the calculations, we need to introduce a regularization cut-off. In our calculation as regularization function we used

$$
C(\Lambda)=\exp \left(-(k / \Lambda)^{4}\right)
$$

where $k$ is the exchanged momentum between the two nucleons. As PC nuclear potential in the Hamiltonian we use the N4LO/N2LO- 500, the N4LO/N2LO-450, and the N4LO/N2LO550 models from Refs. [25, 26], where the final number is the value of the cut-off $\Lambda$ that we matched also for the $\mathrm{CP}$-violating part.

We also evaluated the errors on the numerical coefficients due to the truncation of the chiral expansion as

$$
\left(\delta a_{i}\right)^{2}=\left(\delta a_{i}^{\mathrm{PC}}\right)^{2}+\left(\delta a_{i}^{C \not P}\right)^{2}+\left(\delta a_{i}^{\psi}\right)^{2},
$$

where $\delta a_{i}^{P C}$ is the error associated to the chiral expansion of the PC potential, $\delta a_{i}^{C P}$ the error associated with the chiral expansion of the $\mathrm{CP}$-violating potential computed following 
Table 1. Values of the numerical coefficients for the ${ }^{2} \mathrm{H}$, the ${ }^{3} \mathrm{H}$ and the ${ }^{3} \mathrm{He}$ in units of $e$ fm $\left(a_{n}\left(a_{p}\right)\right.$ in units of $d_{n}\left(d_{p}\right)$ ) for a choice of the cutoff $\Lambda=500 \mathrm{MeV}$.

\begin{tabular}{ccrr} 
& \multicolumn{1}{c}{${ }^{2} \mathrm{H}$} & \multicolumn{1}{c}{${ }^{3} \mathrm{H}$} & \multicolumn{1}{c}{${ }^{3} \mathrm{He}$} \\
\hline$a_{n}$ & $0.939 \pm 0.001$ & $-0.033 \pm 0.001$ & $0.908 \pm 0.009$ \\
$a_{p}$ & $0.939 \pm 0.001$ & $0.909 \pm 0.009$ & $-0.033 \pm 0.001$ \\
$a_{0}^{V}$ & --- & $-0.055 \pm 0.013$ & $0.056 \pm 0.013$ \\
$a_{1}^{V}$ & $0.192 \pm 0.006$ & $0.154 \pm 0.004$ & $0.155 \pm 0.004$ \\
$a_{2}^{V}$ & --- & $-0.120 \pm 0.009$ & $0.118 \pm 0.009$ \\
$a_{\Delta}^{V}$ & $-0.306 \pm 0.174$ & $-0.388 \pm 0.101$ & $-0.383 \pm 0.102$ \\
$A_{1}^{V}$ & --- & $0.006 \pm 0.001$ & $-0.006 \pm 0.002$ \\
$A_{2}^{V}$ & --- & $-0.010 \pm 0.003$ & $0.010 \pm 0.003$ \\
$A_{3}^{V}$ & $0.013 \pm 0.004$ & $-0.008 \pm 0.002$ & $-0.008 \pm 0.002$ \\
$A_{4}^{V}$ & $-0.013 \pm 0.004$ & $0.013 \pm 0.004$ & $0.013 \pm 0.004$ \\
$A_{5}^{V}$ & --- & $-0.022 \pm 0.006$ & $0.022 \pm 0.006$
\end{tabular}

Ref. [27] and $\delta a_{i}^{\psi}$ is the error associated to the numerical accuracy of the 3-body wave function which we estimated to be of the order of $\sim 1 \%$. The calculations of the wave functions were performed using the Hyperspherical Harmonics approach [28].

In Table 1 as an example we report the results obtained for the $a_{i}^{V}$ coefficients and $a_{n}$ and $a_{p}$ computed for a cut-off of $\Lambda=500 \mathrm{MeV}$ for the deuteron, the tritium and the ${ }^{3} \mathrm{He}$. The detailed tables with all the coefficients for all the different cutoffs can be found in Ref. [16].

\section{Conclusions}

In this proceeding we presented the calculation of the EDMs of nuclei within $\chi$ EFT. We discussed the derivation of the CP-violating nuclear interaction with particular attention on the necessity of overcoming the Weinberg's power counting by adding the CP-violating counterterm at LO in order to reabsorb the divergences generated by the OPE in the Schrödinger equation. We also presented a preliminary analysis of the diagrams that contribute to the CP-violating charge operator up to NLO.

Finally we reported some numerical results for which we computed the errors coming from the chiral expansion truncation. The calculation is for now limited on considering contributions to the EDM that come only from the CP-violating nuclear potential. However, the contribution coming from the CP-violating charge density is fundamental in order to have a full consistent description of the nuclear EDMs within $\chi$ EFT. This work is already ongoing.

To conclude we want to remark that the calculations of this dynamical coefficients together with possible future experimental results on EDMs of light nuclei will permit to determine the LECs and then to individuate the sources of CP-violation at the fundamental level.

\section{Acknowledgments}

This work is supported by the U.S. Department of Energy, Office of Nuclear Science, under contracts DE-AC05-06OR23177 (A.G.).

\section{References}

[1] N. Yamanaka, B. Sahoo, N. Yoshinaga, T. Sato, K. Asahi, B. Das, Eur. Phys. J. A 53, 54 (2017), 1703.01570 
[2] T. Chupp, P. Fierlinger, M. Ramsey-Musolf, J. Singh, Rev. Mod. Phys. 91, 015001 (2019), 1710.02504

[3] C.Y. Seng, Phys. Rev. C 91, 025502 (2015), 1411.1476

[4] Y. Yamaguchi, N. Yamanaka, Phys. Rev. Lett. 125, 241802 (2020), 2003.08195

[5] B. Graner, Y. Chen, E. Lindahl, B. Heckel, Phys. Rev. Lett. 116, 161601 (2016), [Erratum: Phys.Rev.Lett. 119, 119901 (2017)], 1601.04339

[6] V. Andreev et al. (ACME), Nature 562, 355 (2018)

[7] C. Abel et al. (nEDM), Phys. Rev. Lett. 124, 081803 (2020), 2001.11966

[8] T. Bhattacharya, V. Cirigliano, R. Gupta, H.W. Lin, B. Yoon, Phys. Rev. Lett. 115, 212002 (2015), 1506.04196

[9] M. Abramczyk, S. Aoki, T. Blum, T. Izubuchi, H. Ohki, S. Syritsyn, Phys. Rev. D96, 014501 (2017), 1701.07792

[10] J. Dragos, T. Luu, A. Shindler, J. de Vries, A. Yousif, Phys. Rev. C 103, 015202 (2021), 1902.03254

[11] E. Mereghetti, W. Hockings, U. van Kolck, Annals Phys. 325, 2363 (2010), 1002 . 2391

[12] J. de Vries, E. Mereghetti, R.G.E. Timmermans, U. van Kolck, The Effective Chiral Lagrangian From Dimension-Six Parity and Time-Reversal Violation (2013), 1212.0990

[13] J. Bsaisou, U.G. Meißner, A. Nogga, A. Wirzba, Annals Phys. 359, 317 (2015), 1412.5471

[14] J. de Vries, R. Higa, C.P. Liu, E. Mereghetti, I. Stetcu, R.G.E. Timmermans, U. van Kolck, Phys. Rev. C84, 065501 (2011), 1109. 3604

[15] J. Bsaisou, J. de Vries, C. Hanhart, S. Liebig, U.G. Meißner, D. Minossi, A. Nogga, A. Wirzba, JHEP 03, 104 (2015), [Erratum: JHEP05,083(2015)], 1411. 5804

[16] A. Gnech, M. Viviani, Phys. Rev. C 101, 024004 (2020), 1906.09021

[17] S. Weinberg, Physica A 96, 327 (1979)

[18] E. Epelbaum, H.W. Hammer, U.G. Meissner, Rev. Mod. Phys. 81, 1773 (2009), 0811. 1338

[19] H.W. Hammer, S. König, U. van Kolck, Rev. Mod. Phys. 92, 025004 (2020), 1906.12122

[20] C. Maekawa, E. Mereghetti, J. de Vries, U. van Kolck, Nucl. Phys. A 872, 117 (2011), 1106.6119

[21] J. Bsaisou, C. Hanhart, S. Liebig, U.G. Meissner, A. Nogga, A. Wirzba, Eur. Phys. J. A 49, 31 (2013), 1209.6306

[22] J. de Vries, A. Gnech, S. Shain, Phys. Rev. C 103, L012501 (2021), 2007.04927

[23] S. Pastore, L. Girlanda, R. Schiavilla, M. Viviani, Phys. Rev. C 84, 024001 (2011), 1106.4539

[24] A. Gnech, R. Schiavilla (2021), to be published

[25] D.R. Entem, N. Kaiser, R. Machleidt, Y. Nosyk, Phys. Rev. C 91, 014002 (2015), 1411.5335

[26] L.E. Marcucci, F. Sammarruca, M. Viviani, R. Machleidt, Phys. Rev. C 99, 034003 (2019), 1809.01849

[27] E. Epelbaum, H. Krebs, U.G. Meißner, Phys. Rev. Lett. 115, 122301 (2015), 1412.4623

[28] L.E. Marcucci, J. Dohet-Eraly, L. Girlanda, A. Gnech, A. Kievsky, M. Viviani, Frontiers in Physics 8, 69 (2020) 